\title{
The Many Faces of Gravity
}

\author{
Robert Sadykov \\ Department of Scientific Research, TATNEFT, Almetyevsk, Russia \\ Email: robertdsadykov@gmail.com
}

How to cite this paper: Sadykov, R. (2020) The Many Faces of Gravity. Journal of High Energy Physics, Gravitation and Cosmology, 6, 817-827.

https://doi.org/10.4236/jhepgc.2020.64054

Received: September 11, 2020

Accepted: October 27, 2020

Published: October 30, 2020

Copyright $\odot 2020$ by author(s) and Scientific Research Publishing Inc. This work is licensed under the Creative Commons Attribution International License (CC BY 4.0).

http://creativecommons.org/licenses/by/4.0/

\begin{abstract}
We show that the number of different gravitational effects is significantly greater than previously thought. First of all, it turned out that the observed constancy of the speed of light relative to the surrounding masses is a special case of a previously unknown fundamental gravitational effect related to the action of gravitation on the speed of light. In other words, the constancy of the speed of light becomes an integral part of gravitation. Moreover, it turned out that the increase in inertial mass and the dilation of the proper time of particles that were accelerated relative to the surrounding masses are also consequences of this fundamental gravitational effect. All of these secondary effects are in the same row with such well-known effects as gravitational refraction and gravitational lensing, which are also a consequence of the action of gravitation on the speed of light. Their belonging to gravitation causes a number of unique features, for example, asymmetry in time dilation and anisotropy of the speed of light, which have been successfully confirmed experimentally. The research is based on a detailed analysis of a large set of experimental data using the classical axiomatic approach.
\end{abstract}

\section{Keywords}

General Relativity, Extended Theories of Gravity, Gravitational Lensing, Cosmology, Background Radiation

\section{Introduction}

All terrestrial experiments on measuring the speed of light in vacuum show the constancy of the local speed of light relative to the Earth. An example of such an experiment is the measurement of the speed of light emitted by accelerated particles. It is clearly seen here that the speed of the emitted light remains constant relative to the Earth and does not add up to the speed of the emitting particles. The results of this experiment are fully consistent with special relativity. It should be noted that the measurement of the speed of light in this experiment was per- 
formed only in the reference frame associated with the Earth. At the same time, the speed of light in the frame of reference of particles remained unknown. The answer to this question was given by the gravitational experiments GRACE and GRACE-FO, the measuring devices of which move at a relatively high speed in a low near-earth orbit [1]. According to general relativity, in the frame of reference of these artificial satellites, which is considered to be inertial in a small local area, the local speed of light should be constant relative to the satellites. However, contrary to expectations, the measured speed of light is still constant relative to the Earth and significantly anisotropic, that is, not constant relative to the satellites themselves. This means that the local speed of light on our planet is always constant relative to our planet, regardless of the choice of the frame of reference. This completely unexpected result is an important turning point that initiates the search for new approaches to relativity and gravitation.

\section{Initial Physical Assumptions}

A remote observer can indirectly observe a decrease in the effective speed of light in the gravitational field of the Sun as a certain delay in the return of the electromagnetic radiation [2]. Taking into account this experimental fact and numerous experimental data that the local speed of light on our planet is constant relative to our planet (see Section 1), we assume the existence of a fundamental gravitational effect that establishes a certain allowed speed of light relative to the source gravitation:

$$
c^{\prime}=c /\left(1+G M / r c^{2}\right),
$$

where $r$ is the distance to mass $M, c$ is the speed of light at an infinite distance, and $G$ is the gravitational constant. This gravitational effect explains the observed constancy of the local speed of light on Earth, as well as other experimental facts that will be considered later, but it explains only half of the delay of the electromagnetic radiation in the gravitational field of the Sun. To explain the second half of the delay, we are forced to assume the existence of a second fundamental gravitational effect that shortens the local length in space around the source of gravitation:

$$
L^{\prime}=L /\left(1+G M / r c^{2}\right),
$$

where $L$ is the unit of length at an infinite distance. Shortening the local unit of length $L^{\prime}$ leads to some increase in the total number of units of length on the trajectory of light (Figure 1).

Due to the lengthening of the trajectory, the remotely observed effective speed of light becomes equal to

$$
c^{\prime \prime}=c^{\prime} L^{\prime} / L=c /\left(1+2 G M / r c^{2}+G^{2} M^{2} / r^{2} c^{4}\right) .
$$

Thus, the delay of electromagnetic radiation observed in the gravitational field of the Sun is a secondary gravitational effect, which is formed by the fundamental gravitational effect of the allowed speed of light and the fundamental gravitational effect of shortening the local length. 


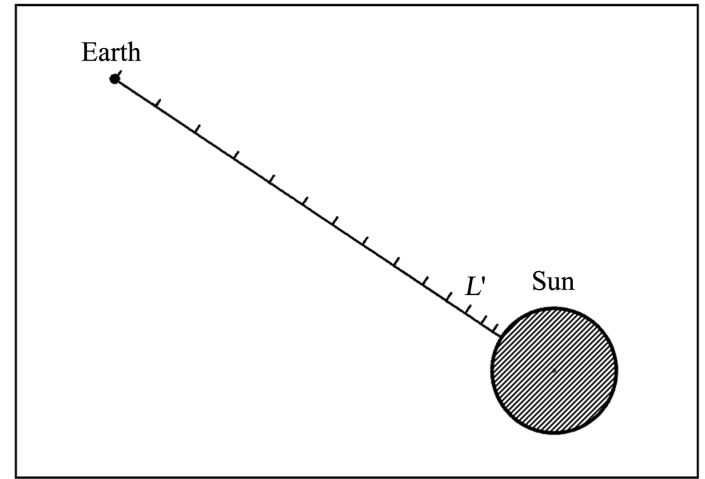

Figure 1. Shortening the local length.

\section{Gravitational Refraction}

Due to the variable effective speed of light $c^{\prime \prime}$ caused by the two fundamental gravitational effects (see Section 2), the gravitational field of central mass $M$ acts as a refracting medium with a variable index of refraction:

$$
n=1+2 G M / r c^{2}+G^{2} M^{2} / r^{2} c^{4} .
$$

The angle of refraction of light in weak gravitational fields, for example, in the gravitational field of the Sun (Figure 2) is equal to

$$
\alpha=4 G M / r c^{2},
$$

where $r$ is the minimal distance of the light ray from the center of mass $M$.

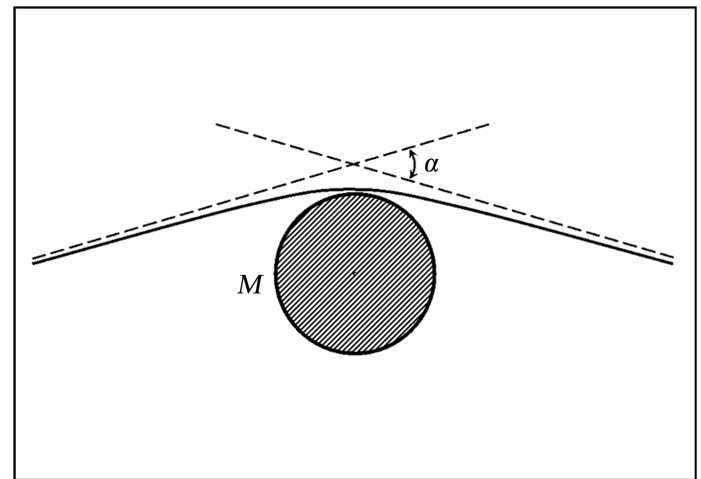

Figure 2. Gravitational refraction.

This angle is two times greater than the Newtonian value for the deflection of light. Thus, the gravitational refraction is a secondary gravitational effect, which is formed by the fundamental gravitational effect of the allowed speed of light and the fundamental gravitational effect of shortening the local length. Gravitational lensing, in turn, is a consequence of gravitational refraction and therefore can be classified as a tertiary gravitational effect.

\section{Gravitational Attraction}

Consider the following physical model. Inside an ideal reflecting sphere, a large number of photons move in different directions forming a spherical photon clus- 
ter. The total kinetic energy of photons forms the internal energy of this cluster $E$. In addition to internal energy, the photon cluster is also characterized by an effective inertial mass:

$$
m=E / c^{2}
$$

Due to the large number of photons and their numerous reflections, this cluster can be viewed as a modified light clock. Thus, the photon cluster as a physical model can simulate the energy, inertial, and temporal properties particles with a non-zero inertial mass. This allows the cluster to act as a test mass.

Let the photon cluster be located in the gravitational field of the central mass $M$ (Figure 3). Gravitational refraction violates the initial dynamics of photons forming the cluster, which leads to acceleration of the cluster in the direction of mass $M$, that is, the gravitational attraction arising under these conditions is a direct consequence of gravitational refraction.

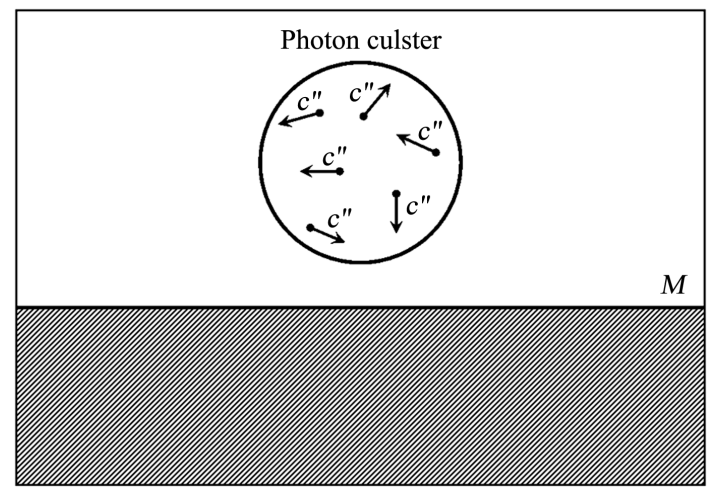

Figure 3. Gravitational attraction.

Gravitational refraction has some features: 1) photons moving in the horizontal direction have the maximum possible refraction in a given local area; 2) refraction is completely absent for photons moving in the vertical direction; 3) other photons have different intermediate refraction. These factors determine the variable dependence of the gravitational acceleration of the photon cluster on the direction and speed of motion of the cluster relative to the central mass $M$. Consider three different cases:

1) When the photon cluster moves in a gravitational field at a speed $v^{\prime \prime}$ much less than the effective speed of light $c^{\prime \prime}$, the gravitational acceleration of the cluster coincides with the classical Newtonian value, regardless of the direction of motion of the cluster;

2) If the photon cluster moves in a gravitational field at a comparatively high speed in the vertical direction, then the gravitational acceleration of the cluster becomes less than the classical value due to a decrease in the refraction of some part of the photons;

3) If the photon cluster moves in a gravitational field in the horizontal direction at a speed $v^{\prime \prime}$ close to the effective speed of light $c^{\prime \prime}$ (Figure 4), then the 
gravitational refraction of most photons approaches the double Newtonian value, and as a result, the gravitational acceleration of the photon cluster also tends to double.

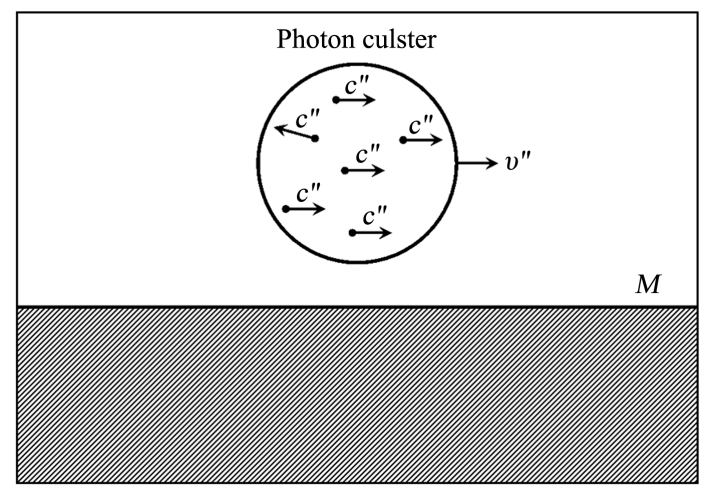

Figure 4. Movement along the horizon.

In practice, the latter case can be observed as the double angle of deflection of the test particle moving relative to the central mass at sublight speed. In the case of a slower motion of the planet Mercury in the gravitational field of the Sun, the increase in gravitational acceleration in comparison with the classical Newtonian value is extremely small, and this is observed as a small precession of its perihelion (Figure 5). The value of the precession increases in stronger gravitational fields along with an increase in numerical value of the horizontal component of speed.

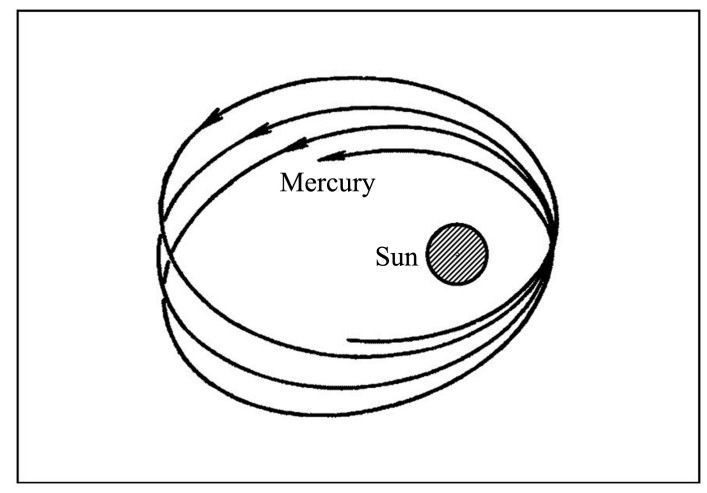

Figure 5. Perihelion precession.

Thus, the gravitational attraction accelerating a photon cluster or any test particle to the central mass is a tertiary gravitational effect based on the fundamental gravitational effect of the allowed speed of light and the fundamental gravitational effect of shortening the local length. Identical gravitational acceleration of various particles [3] under the same initial conditions means that the internal energy of these particles, as in the case of the photon cluster, is contained in the form of kinetic energy. In other words, any potential energy, including the internal energy of particles, is a latent form of kinetic energy. 


\section{Gravitational Time Dilation}

Let the remote observer located at point $A$ (Figure 6). The photon cluster moves from point $A$ to point $B$ located on the surface of the central mass $M$.

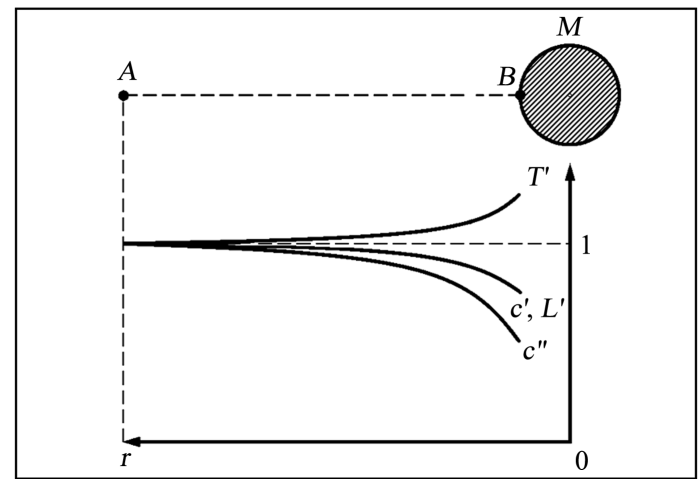

Figure 6. Gravitational time dilation.

A decrease in the allowed speed of photons $c^{\prime}$ relative to the central mass $M$ (see Section 2) leads to an increase in the period between reflections of photons, which can be considered as a dilation of the proper time of the cluster:

$$
T^{\prime}=T c / c^{\prime}=T\left(1+G M / r c^{2}\right) \text {. }
$$

The same thing happens with an ordinary light clock after moving from point $A$ to point $B$.

If we add in the considered physical model the fundamental gravitational effect of shortening the local length, then the remotely observed effective speed of light at point $B$ decreases to a value of $c^{\prime \prime}$ (see Section 2), but this does not lead to an additional dilation of the proper time of the photon cluster or the light clock, because all geometric dimensions of these devices decrease in three spatial dimensions in proportion to the shortening of the local length. Thus, the gravitational time dilation is a secondary gravitational effect, which is formed only by the fundamental gravitational effect of the allowed speed of light.

It is important to note that the allowed speed of light $c^{\prime \prime}$ and the effective speed of light $C^{\prime \prime}$ are related to remote observation and only to it. From the point of view of the local observer located at point $B$, the local speed of light has a typical value of $c$. The reason for this lies in the local peculiarities of speed measurement: during a unit of local time dilated by the gravitational effect of the allowed speed of light, the photon slowed down by this gravitational effect travels a local distance typical of light.

\section{Gravitational Energy}

The internal energy of the photon cluster consists of the kinetic energy of photons that form the cluster (see Section 4). Gravitational acceleration of the photon cluster (Figure 3 ) is accompanied by the transformation of the internal energy of the cluster into the external kinetic energy. Gravitational refraction initiates 
this transformation, but like any other refraction does not change the total energy of the system. As a result, the internal energy of the cluster decreases:

$$
E^{\prime}=E /\left(1+G M / r c^{2}\right) \text {, }
$$

but the total energy remains constant. This conclusion obtained for the photon cluster can be extended to any test particle with a nonzero inertial mass due to their inertial, energy, temporal, and gravitational equivalence (see Section 4). Thus, contrary to expectations, the gravitational energy is not contained in the gravitational field. Instead, gravitation uses the internal energy of the test particle to gravitational acceleration this particle.

Given the properties of gravitation discussed above, the remote observer located at point $A$ (Figure 6) can interpret the decrease in the frequency of light $f^{\prime}$ arriving from point $B$ as a consequence of the gravitational dilation of the proper time of the light source $T^{\prime}$ :

$$
f^{\prime}=f T / T^{\prime}=f /\left(1+G M / r c^{2}\right) \text {. }
$$

In addition, he can interpret this as a consequence of a decrease in the internal energy of the light source $E^{\prime}$. Conversely, a locally observed increase in the frequency and energy of light arriving at point $B$ from point $A$ can be interpreted as a gravitational dilation of the proper time and a decrease in the intrinsic energy of the local observer. In other words, the gravitational redshift and gravitational blueshift are a consequence of the action of gravitation on the basic physical characteristics of the radiation source and local observer, but not the action of gravitation on the frequency and energy of electromagnetic radiation.

\section{Two Different Forms of Gravitational Time Dilation}

The gravitational effect of the allowed speed of light dilates the proper time of the light clock $T^{\prime}$ at point $B$ (Figure 6) by decreasing the allowed speed of light $c^{\prime}$ relative to the central mass $M$ (see Section 5). When the same light clock accelerates to speed $v^{\prime}$, this gravitational effect still maintains the allowed speed of light $c^{\prime}$ relative to the central mass, which increases the period of passage of the photon between the mirrors of the light clock (Figure 7).

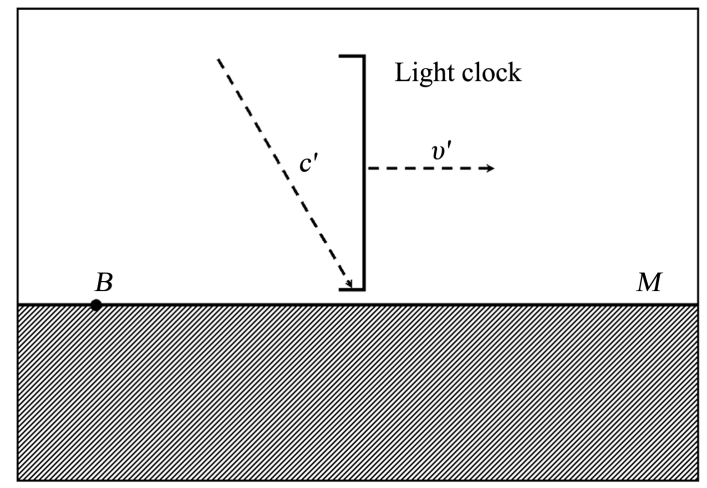

Figure 7. The second form of gravitational time dilation. 
This leads to an additional dilation of the proper time of this clock:

$$
T^{\prime \prime}=T^{\prime} / \sqrt{1-v^{\prime 2} / c^{\prime 2}} \text {. }
$$

A similar result is also obtained when the light clock is replaced with a photon cluster. In addition to time dilation, the acceleration of the photon cluster leads to an increase in the total energy of photons, which is accompanied by an increase in the inertial mass of the cluster:

$$
m^{\prime}=m / \sqrt{1-v^{\prime 2} / c^{\prime 2}} .
$$

Thus, time dilation is a consequence of the fundamental gravitational effect of the allowed speed of light and is realized in two slightly different forms: the first form $T^{\prime}$ appears when the test particle is placed in the vicinity of the source of gravitation; the second form $T^{\prime \prime}$ arises when the test particle moves relative to the source of gravitation. Both forms of time dilation take place on the satellites of the Global Positioning System [4].

\section{Propagation of Light in a Moving Medium}

Let the remote observer located at point $A$ (Figure 6). The transparent cube moves at a speed of $v^{\prime}$ in the vicinity of the central mass $M$. By condition, the speed $v^{\prime}$ is much less than the allowed speed of light $c^{\prime}$ in this local area. The photon moves inside the transparent cube in the same direction as the cube. From time to time the photon is absorbed and after a short time is again emitted by the particles of the cube. The conditional speed of the photon after each absorption by particles is equal to the speed of the cube $v^{\prime}$. When the photon is re-emitted and moves between particles, its speed relative to the central mass $M$ is equal to the allowed speed of light $c^{\prime}$. As a result, the average speed of the photon becomes equal to

$$
c_{2}=c_{1}+v^{\prime}\left(1-\left(c_{1} / c^{\prime}\right)^{2}\right),
$$

where $c_{1}$ is the average speed of the photon when the speed of the cube is zero. This result, known as the partial dragging of light, was observed in the water flow in the Fizeau experiment [5]. Thus, the Fizeau optical experiment is another convincing proof of the existence of the allowed speed effect.

\section{Feature of Gravitation in Interstellar Space}

Consider the following physical model. The photon moves from remote point $A$ to point $B$ located in the central hollow area of the spherically symmetric mass $M$ (Figure 8). In the process, the fundamental gravitational effect of the allowed speed of light gradually decreases the allowed speed of the photon $c^{\prime}$ (see Section 2). In addition to this, the fundamental gravitational effect of shortening the local length reduces the effective speed of the photon $c^{\prime \prime}$ (see Section 2). This speed reaches a minimum in the empty central area (see Diagram in Figure 8). Mass $M$ from this area can be considered as some system of surrounding masses. One of several secondary gravitational effects-gravitational refraction (see Sec- 
tion 3) is completely absent here due to the constant effective speed of light $c^{\prime \prime}$ in the entire central area. The absence of gravitational refraction, in turn, leads to the absence of tertiary gravitational effects-gravitational lensing and gravitational attraction (see Sections 3 and 4). However, other derivative gravitational effects and two fundamental gravitational effects continue to act. In particular, the internal energy of the test particle $E$ (see Section 6) moved from point $A$ to the central area decreases to a value of $E^{\prime}$ (see Diagram in Figure 8). Parallel to this, the proper time of the test particle $T$ dilates to a value of $T^{\prime}$ (see Section 5) and additionally dilates to a value of $T^{\prime \prime}$ in the case of the motion of the test particle relative to mass $M$ (see Section 7). Due to the gravitational effect of shortening the local length, the geometric dimensions of the test particle decrease in all three spatial dimensions in proportion to the shortening of the local length. However, this decrease goes unnoticed in local observation due to the identical action of gravitation on the observer himself. Due to the gravitational effect of the allowed speed of light, the speed of light $c^{\prime}$ in the central area remains constant relative to mass $M$, even if the light is emitted by a moving particle. In the case of local observation, this speed has a typical value of $c$ (see Section 5).

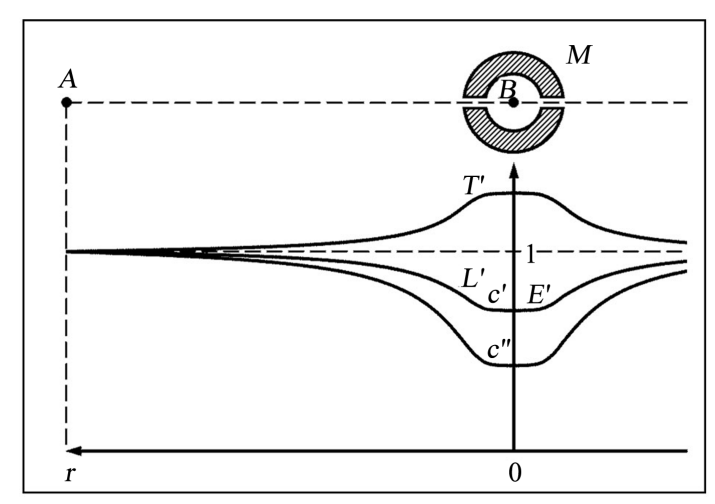

Figure 8. Spherically symmetric hollow mass.

The same physical conditions are created in interstellar space. This means that the constant speed of light propagating from moving space objects, such as binary stars or astrophysical jets, is a consequence of the fundamental gravitational effect of the allowed speed of light, which establishes the allowed speed of light relative to the system of surrounding masses. The dilation of the proper time of various cosmic particles accelerated relative to the system of surrounding masses is also a consequence of this gravitational effect. Particles moving in different directions but at the same speed relative to the system of surrounding masses have an identical time dilation. In the frames of reference of these particles, the proper time of the surrounding stars flows faster than usual in proportion to the dilation of the proper time of the particles. Due to the fixation of the allowed speed of light relative to the system of surrounding masses, stellar aberration is observed only when the observer moves relative to this entire system. If instead, the observed star moves relative to the system of surrounding masses, then stel- 
lar aberration is absent. Thus, the high constancy of the speed of light from astrophysical jets and binary stars, the violation of symmetry in time dilation, and the feature of observation of stellar aberration are very strong evidence of the existence of the gravitational effect of the allowed speed of light.

\section{Gravitation and Background Radiation}

The distant areas of the Universe in which the cosmic microwave background radiation observed today was formed are moving away from us at a superluminal speed. The relic electromagnetic radiation from these areas, which moves in our direction, should also move away from us, and this really took place at the very beginning. However, the fundamental gravitational effect of the allowed speed of light acts along the entire path of propagation of electromagnetic radiation. As a result, on the scale of the Universe, the speed of relict photons changes over time, but in each specific area of the Universe, photons move at the allowed speed of light $c^{\prime}$ relative to the comoving system of surrounding masses (see Section 9) and ultimately reach us. Thus, the observation of the background radiation once again confirms the presence of the gravitational effect of the allowed speed of light.

\section{Experimental Confirmation}

The existence of the fundamental gravitational effect of shortening the local length is indirectly confirmed by four experimental facts: gravitational time delay, gravitational refraction, gravitational lensing, and finally gravitational attraction (see Sections 2 - 4).

The fundamental gravitational effect of the allowed speed of light is directly and indirectly confirmed by a very large number of experimental data (see Sections $1-10$ ), including the constancy of the local speed of light relative to the Earth (see Section 1). Additional confirmation of this gravitational effect is the Sagnac experiment [6], which again shows the constancy of the local speed of light relative to the Earth. This is especially evident in the version of the experiment with the propagation of light around the Earth. The high-precision work of the Global Positioning System also demonstrates the constancy of the local speed of light relative to the massive Earth and the noticeable anisotropy of the speed of light in the reference frame related to the satellites. The difference in the speeds of light on these satellites along their orbit is equal to double orbital speed. As the relative speed increases, the numerical value of the anisotropy of the speed of light should increase accordingly, which is reliably confirmed by satellites GRACE and GRACE-FO (see Section 1). Thus, the gravitational experiments of the GRACE series are the key experiments confirming the existence of the gravitational effect of the allowed speed of light.

\section{Conclusion}

Against the background of many modern physical theories, general relativity is 
deservedly considered the most advanced physical theory. At the same time, special relativity is the most consistent physical theory. These theories very accurately reflect reality in all existing parallel universes, except for one atypical universe. Our Universe is such an exception. In our unique Universe, gravitation differs significantly from acceleration; in particular, the free fall of a test particle is accompanied by a decrease in the internal energy of this particle (see Section 6). This does not happen with any non-gravitational acceleration. In our amazing Universe, the constancy of the speed of light, the increase in inertial mass, and two slightly different forms of time dilation are derivative gravitational effects (see Sections 7 and 9). In our unusual Universe, gravitational attraction is a tertiary gravitational effect (see Section 4). Unfortunately, in our Universe, there is no longitudinal length contraction, but there is a vaguely similar gravitational analog that really shortens the length in all three spatial dimensions (see Sections 2 - 5 and 9).

\section{Conflicts of Interest}

The author declares no conflicts of interest regarding the publication of this paper.

\section{References}

[1] Yan, Y.H., Muller, V., Heinzel, G. and Zhong, M. (2020) Revisiting the Light Time Correction in Gravimetric Missions Like GRACE and GRACE Follow-On. https://doi.org/10.5194/gstm2020-4

[2] Shapiro, I.I., Pettengill, G.H., Ash, M.E., Stone, M.L., Smith, W.B., Ingalls, R.P. and Brockelman, R.A. (1968) Fourth Test of General Relativity: Preliminary Results. Physical Review Letters, 20, 1265-1269. https://doi.org/10.1103/PhysRevLett.20.1265

[3] Eötvös, R.V. (1890) Mathematische und Naturwissenschaftliche Berichte aus Ungarn. 8, 65.

[4] Neil, A. (2002) Relativity in the Global Positioning System. Physics Today, 55, 45. https://doi.org/10.1063/1.1485583

[5] Hippolyte, F. (1851) The Hypotheses Relating to the Luminous Aether, and an Experiment Which Appears to Demonstrate That the Motion of Bodies Alters the Velocity with Which Light Propagates Itself in Their Interior. Philosophical Magazine, 2, 568-573. https://doi.org/10.1080/14786445108646934

[6] Sagnac, G. (1913) L'ether lumineux demontre par l'effet du vent relatif d'ether dans un interferometre en rotation uniforme. Comptes Rendus, 157, 708-710. 